See discussions, stats, and author profiles for this publication at: https://www.researchgate.net/publication/281161552

\title{
Molecular model for hydrated biological tissues
}

Article in Physical Review E · June 2015

DOl: 10.1103/PhysRevE.91.063310

\section{CITATIONS}

5 authors, including:

\section{Érika Tiemi Sato}

4 PUBLICATIONS 28 CITATIONS

SEE PROFILE

2. Luis Felipe Das Chagas e Silva de Carvalho

Universidade de Taubaté

40 PUBLICATIONS 141 CITATIONS

SEE PROFILE

\section{READS}

114

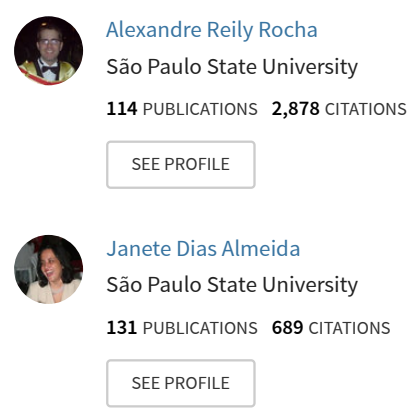

Some of the authors of this publication are also working on these related projects:

Water/Metal Interfaces View project

Computational simulation of TiO2-based memristive systems: from the raw material to the device View project 


\title{
Molecular model for hydrated biological tissues
}

\author{
Erika Tiemi Sato, ${ }^{1}$ Alexandre Reily Rocha, ${ }^{2}$ Luis Felipe das Chagas e Silva de Carvalho, ${ }^{3}$ \\ Janete Dias Almeida, ${ }^{4}$ and Herculano Martinho ${ }^{1, *}$ \\ ${ }^{1}$ Universidade Federal do ABC, Av. dos Estados, 5001, Bangu, 09210-580, Santo André-SP, Brazil \\ ${ }^{2}$ Instituto de Física Teórica, Universidade Estadual Paulista (UNESP), R. Dr. Bento Teobaldo Ferraz, 271 - Bloco II, Barra-Funda, \\ 01140-070, São Paulo-SP, Brazil \\ ${ }^{3}$ FOCAS Institute, Dublin Institute of Technology, Camben Row, Dublin 8, Ireland \\ ${ }^{4}$ Depto. de Biociências e Diagnóstico Bucal, Instituto de Ciência e Tecnologia, Campus São José dos Campos, \\ Universidade Estadual Paulista (UNESP), Av. Eng. Francisco José Longo, 777, Jardim São Dimas, 12245-000, \\ São José dos Campos-SP, Brazil \\ (Received 28 October 2014; published 25 June 2015)
}

\begin{abstract}
A density-functional microscopic model for soft tissues (STmod) is presented. The model was based on a prototype molecular structure from experimentally resolved type I collagen peptide residues and water clusters treated in periodic boundary conditions. We obtained the optimized geometry, binding and coupling energies, dipole moments, and vibrational frequencies. The results concerning the stability of the confined water clusters, the water-water, and water-collagen interactions were successfully correlated to some important experimental trends of normal and inflammatory tissues.
\end{abstract}

DOI: 10.1103/PhysRevE.91.063310

PACS number(s): 31.15.A-, 87.14.em, 87.17.Aa, 87.64.kp

\section{INTRODUCTION}

The role of water in biological systems is still, in many cases, an enigma even though this molecule is an integral part of many biomolecular complexes. In particular, proteins have much of their properties governed by interactions with water [1]. Leikin et al. [2] have shown that hydration force effects resulting from the energetic cost of water rearrangements near a collagen surface display distinguished features in its Raman spectra. We have shown, in a previous study [3], that water behaves differently in healthy and pathological tissues. Vibrational modes analysis compared to literature results [4,5] showed that the inflammatory process led to changes in the high-wave number spectral region related to collagen and confined water. Furthermore, intracellular hydration can be of great relevance in cancerous processes, since cancer cells contain more free water than normal cells. The cellular hydration increases with increasing degree of malignancy [6,7]. Hydropic degeneration (excess of water absorption by the epithelial tissue) and biochemical redox reactions are two important characteristics of the inflammatory process.

The prevalence of typical water clusters in inflammatory tissues is a known event reported in the literature [8]. Cibulsky and Sadlej [5] observed that the $\mathrm{OH}$-stretching vibrations region of Raman spectra are related to local structures and interactions of the hydrogen-bond networks, so each water cluster has a characteristic Raman spectrum. de Carvalho et al. [3] pointed out that this could be the origin of the discriminating power of the high-wave number region closely related to the hydropic degeneration process in inflammatory fibrous hyperplasia. Raman spectroscopy studies performed by Gniadecka et al. [9] found an increased amount of the nonmacromolecule bound, tetrahedral water in photo-aged and malignant tumors of the skin compare to normal tissues. These experimental results indicated the relevance of understanding the role of electrons, protons, and hydration water in tissues.

\footnotetext{
*herculano.martinho@ufabc.edu.br
}

Computational simulations are widely used to study and make predictions concerning a broad variety of systems ranging from pharmacology to engineering fields [10-12]. Atomistic models based on quantum mechanics calculations have the greatest predictive capability for materials properties. However, due to their inherent complexity (aperiodicity and large number of atoms), detailed atomistic models for biological tissues are absent in the literature. Such models would be useful to understand physical and biochemical properties of tissues. For example, little is known about the underlying mechanisms of cell migration in wound healing, specially the modulating role of mechanical tension at the micro-environmental scale [13]. Usually finite-element classical approaches have been applied to simulate mechanical characteristics of soft tissues such as skin [14]. However, many physical properties have not been satisfactorily simulated by these conventional models $[15,16]$. Monte Carlo methods have been generally considered as the standard to simulate light propagation in heterogeneous tissues and to validate the results obtained by other models. The main drawbacks of this method are the extensive computational burden [15], absence of realistic phase function, and elastic light scattering models [16]. Moreover, the explicit structure of water and its interactions with the neighbors are not accounted for.

The dynamics of atoms, molecules, electrons, protons, and hydration water using quantum mechanical approaches, to the best of our knowledge, have only been studied for isolated or solvated biomolecules. Simulation models reported are mostly classical [17-20] or hybrid models [21]. Of all proteins present in our body, collagen is the most abundant and for this reason has been exhaustively studied. An atomistic model based on this important biomolecular complex would enable the study of the molecular interactions which would be present; to quantify the relative strength of each interaction; to perform dynamical simulations; and to predict properties as, e.g., electronic structure, transport, and absorption, emission, and vibrational spectra with high degree of accuracy. To correctly describe soft tissues and be computationally viable 
the atomistic model should be small enough and include water-water and water-molecule effects.

In the present work a microscopic model for soft biological tissue based on Density Functional Theory (DFT) is presented. This model, named Soft Tissue model (STmod) retains some fundamental atomic connectivity based on the collagen fibrils constitution. However, it is important to stress that STmod is not a simple solvating collagen model. The stability of some proposed variations of the model was studied, and compared to relevant experimental results.

\section{METHODOLOGY}

Figure 1(a) shows a general scheme of a real connective soft tissue. The different collagen types form large fibrillar bundles. These collagen fibrils are semi-crystalline aggregates of collagen molecules and water ( $>1000$ amino acids). Approximately $60 \%$ of the dry tissue has proteic constitution (collagen fibrils) [22]. Figure 1(b) shows the dimension scale of STmod. In order to built the STmod we associated the set of collagen fibers to a reticulated set of unit cells whose internal constitution should be appropriately chosen. In our approximation the complexity of the real tissue was replaced by this periodic set using periodic boundary conditions (PBC). PBC are usually chosen for approximating a very large (or infinite) system by using a small part of it (unit cell) for theoretical modeling of both periodic or aperiodic systems. Considering typical collagen fibers dimensions and the relative composition of $\sim 60 \%$ of fibers in the tissue, one could estimate typical tissue is composed by $\sim 10^{12} \mathrm{STmod}$ unit cells $/ \mathrm{mm}^{2}$. The PBC approximation could be justified by this estimate. The STmod unit cell was built starting with an experimentally resolved hydrated collagen type I structure obtained from the Protein Data Bank [23]. A specific peptide sequence of this structure was cut retaining proline, glycine, and hydroxyproline amino acids which enclosed some water molecules. An example of structure enclosing four water molecules is shown in Fig. 1(c). The criteria used to choose this specific peptide sequence were based on (i) the presence of water molecules confined in the original structure; (ii) the presence of residues of amino acids; (iii) the viable number of amino acids with respect to time of calculation (typically $\sim 100$ atoms). Two variants of this small unit were chosen (labeled $C$ and $D$ structures). The $C$ structure has orthorhombic symmetry ( $P 2_{1} 2_{1} 2_{1}$ space group); unit cell parameters $a=13.5 \AA, b=11.5 \AA$, and $c=10.5 \AA$, and internal cage volume (oblate spheroid) $V_{c}=1,857.3 \AA^{3}$ (see Table I). The corresponding dry structure $C_{0}$ is show on the left-hand side of Fig. 2. To build several hydrated models, an specific water clusters were inserted in the center of the structure. Water cluster structures $\left(\mathrm{H}_{2} \mathrm{O}\right)_{n}(1 \leqslant n \leqslant$ $8)^{1}$ were chosen based on the TIP4P model [24] from the Cambridge Cluster Database [25]. Therefore, we label the STmod structures $C_{n}(0 \leqslant n \leqslant 8)$. The effect of the presence of an external hydration shell was checked on the $C_{1}$ structure.

\footnotetext{
${ }^{1}$ It is possible the existence of more than a single symmetry for the same atomic set for $n \geqslant 5$. Thus, we followed the choice of [5] and considered only the structures $\left(\mathrm{H}_{2} \mathrm{O}\right)_{5 \text {-cyclic }},\left(\mathrm{H}_{2} \mathrm{O}\right)_{6 \text {-cage }},\left(\mathrm{H}_{2} \mathrm{O}\right)_{7 \text {-low }}$, and $\left(\mathrm{H}_{2} \mathrm{O}\right)_{8-S 4}$.
}
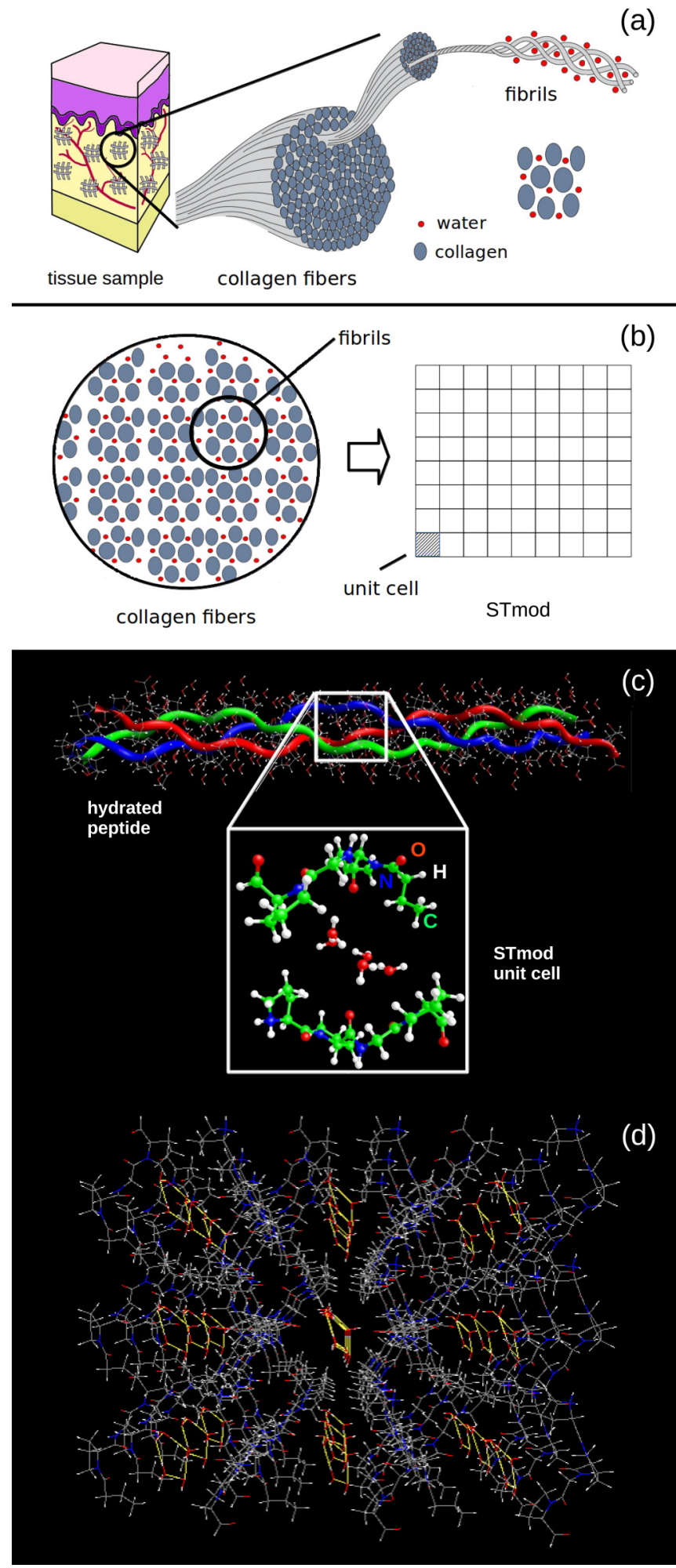

FIG. 1. (Color online) (a) Structural scheme of a connective tissue showing the collagen fibers and the fibrils. These are constituted by water and collagen molecules. (b) The construction of the STmod unit cell compared the original collagen fibers structure. (c) STmod unit cell example $\left(C_{4}\right.$ structure) chosen from a experimentally resolved hydrated peptide structure. (d) $C_{4}$ structure enclosing four water molecules clusters. In (c) and (d) hydrogen bond inside water clusters were indicated by yellow. Hydrogen, carbon, nitrogen, and oxygen atoms were in white, green, blue, and red, respectively. 
TABLE I. Parameters for simulated structures. The space group was $P 2{ }_{1} 2_{1} 2_{1}$ (orthorhombic) in all cases. The unit cell lattice parameters $(a, b, c)$, the cage volume $\left(V_{c}\right)$, the number of internal (cage), and external water molecules are also shown.

\begin{tabular}{lccc}
\hline \hline parameter & $C_{n}$ & $C_{1 s}$ & $D_{0}, D_{1}$ \\
\hline$a(\AA)$ & 13.5 & 15.0 & 17.4 \\
$b(\AA)$ & 11.5 & 12.5 & 13.3 \\
$c(\AA)$ & 10.5 & 20.0 & 9.8 \\
$V_{c}=\left(\AA^{3}\right)$ & $1,857.3$ & $1,857.3$ & 182.8 \\
cage waters & $0<n<8$ & 1 & 1 \\
external waters & 0 & 10 & 0 \\
\hline \hline
\end{tabular}

Ten external water molecules were added to the $C_{1}$ structure $\left(C_{1 s}\right.$ structure) shown in the center of Fig. 2. Another structural variant is the compact version of $C$ structure, with unit cell parameters $a=17.4 \AA, b=13.3 \AA, c=9.8 \AA$, and $V_{c}=$ $182.8 \AA^{3}$. Only dry ( $D_{0}$, right of Fig. 2) and monohydrated $\left(D_{1}\right)$ structures were considered in the present study. Each cell was replicated [see Fig. 1(d), for example] in order to describe the soft tissue. It is important to stress that $C_{n}, C_{1 s}$, and $D_{1}$ structures represent the most common sites where water clusters could be present in fibrils and collagen [23,26,27].

Molecular mechanics models are useful for simulations of conformal energies and non-covalent interactions of complex molecular systems [28]. The MMFF94s force field [29] uses the van der Waals term and includes cubic and quartic terms in the bond stretch, and cubic terms in angle bending potential energy. MMFF94s produces relatively flat dynamically averaged structures [29]. The $C_{n}$ models were pre-optimized in a first step using molecular mechanics with the MMFF94s force field [29] implemented in the Avogadro software [30]. The $D$ models were optimized in a first step using Hartree Fock (HF) with the 6-31 $+g(2 d)$ basis implemented in the Gaussian package [31]. HF methods are one of the least computationally demanding quantum methods.

Finally, Density Functional Theory (DFT) [32,33] was used in order to obtain the equilibrium geometries and harmonic frequencies. The convergence of the calculation was also checked by computing the total energy as a function of unit cell volume variation. We notice that a converged energy value (99.2\% of the initial value) was found for $5 \%$ unit cell volume variation. The confinements were implemented in the CPMD program [34] using the BLYP functional [35] augmented with dispersion corrections for the proper description of van der Waals interactions [36,37]. For all simulations, the cutoff

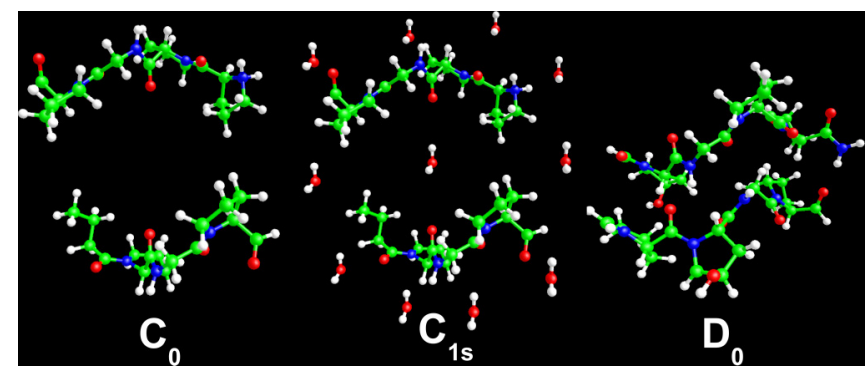

FIG. 2. (Color online) $C_{0}, C_{1 s}$, and $D_{0}$ structures. energy was considered up to $100 \mathrm{Ry}$. The wave functions were optimized and then the vibrational modes were obtained with Raman responses using the Hessian matrix. Finally, the linear response for the values of polarization and polar tensors of each atom in the system was calculated to evaluate the eigenvectors of each vibrational mode. Harmonic frequencies were compared to experimental Raman data for normal (NM) and inflammatory (IFH) oral mucosa tissue (see Ref. [3] for experimental details). The spectra were simulated as a convolution of Gaussian lineshape peaks centered on the calculated frequencies. The linewidth was chosen to be $20 \mathrm{~cm}^{-1}$.

\section{RESULTS AND DISCUSSION}

$C_{0}$-normalized total energies for each structure are shown in Fig. 3(a). We compared the energies of non-optimized and geometry optimized structures. Since the non-optimized structures retained the original experimental structural parameters, this comparison is of interest. In all structures the total energy decreased by less than $20 \%$ with an increase in the difference, the greater the water cluster size was. The results show that a larger number of molecules per cluster lowers the cluster energy. An exponential dependence on water content,
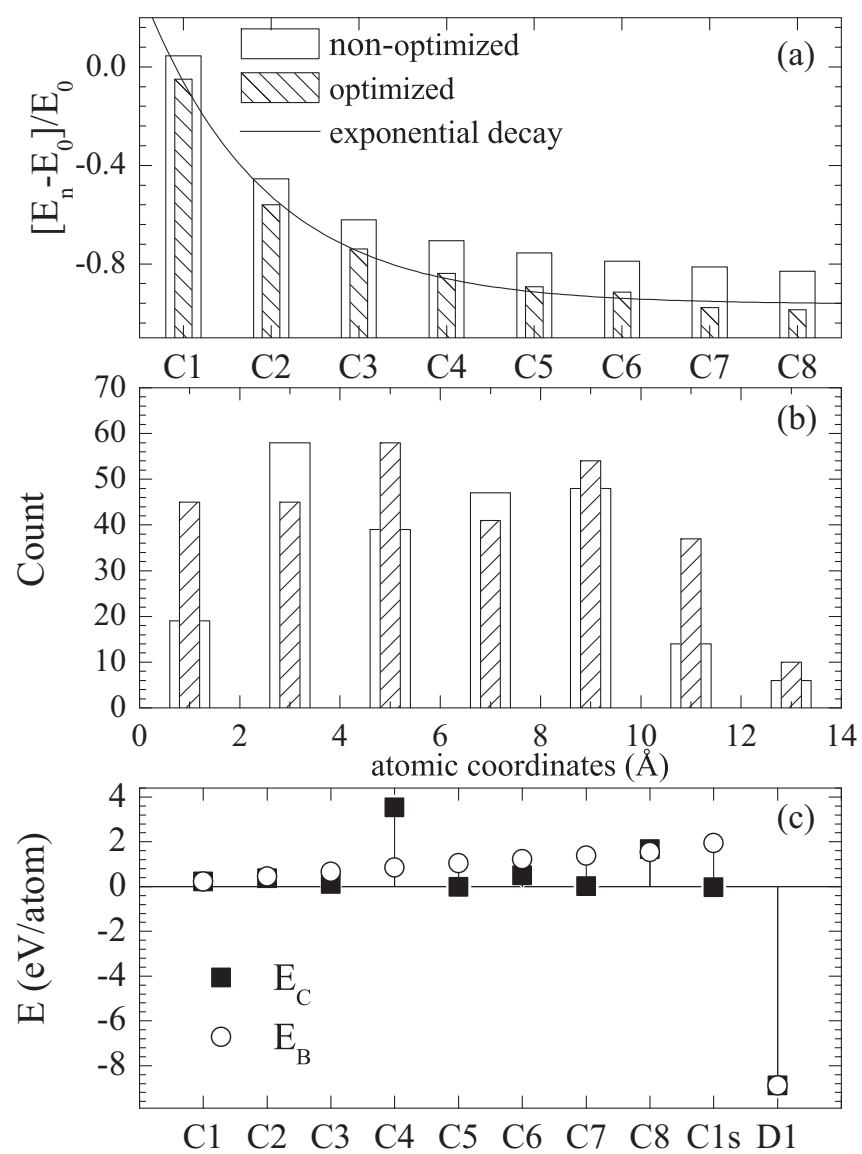

FIG. 3. (a) Normalized total energy per atom for optimized and non-optimized $C$ structures. (b) Histogram for atomic coordinates of $C_{0}$ showing the effect of geometry optimization. (c) Binding $E_{B}$ (open circle) and coupling $E_{C}$ (closed square) energies as defined in Eqs. (2) and (3), respectively, for the various structures. 
$n$, according to

$$
\frac{E_{n}-E_{0}}{E_{0}}=-0.964+1.86 e^{\left(-\frac{n}{1.388}\right)}
$$

was observed [solid line in Fig. 3(a)]. It is important to notice that all cluster structures were stable $\left(E_{n}<E_{0}\right)$. Park et al. [38] analyzed structures and energies of the dimer to heptamer water clusters using the revised empirical potential function for conformational analysis. They realized that the cyclic structures for water clusters are more favorable than open structures in general, except for the heptamer case. They also noted the more hydrogen bonds a cluster has, the greater its stability. Thus, the stability of the chain of clusters increases by adding a water molecule to the cluster. However the energetic gain for $C_{4}<C<C_{8}$ was $\lesssim 8 \%$. It could be stated the energetic gain was minimum for $C>C_{4}$.

Figure 3(b) shows a histogram of atomic distances for non-optimized and optimized $C_{0}$ structures in order to compare the effect of optimization in the experimentally determined original atomic positions. The observed tendency was a small displacement at $\lesssim 5 \%$ from their original arrangements. However, it is important to stress that the original atomic connectivity and symmetry remain unchanged.

Characterization of the inter-water molecule interactions, and the interactions between confined water molecules and collagen clusters was done by calculating the binding energy $\left(E_{B}\right)$ and the coupling energy $\left(E_{C}\right)$, respectively. $E_{B}$ is defined by

$$
E_{B}=E_{\text {STmod }}-\left(E_{0}+n E_{\mathrm{H}_{2} \mathrm{O}}\right),
$$

and measures the average interaction between the free water molecules and their environment. $E_{\mathrm{STmod}}, E_{0}$, and $E_{\mathrm{H}_{2} \mathrm{O}}$ are STmod unit cell, $C_{0}$ or $D_{0}$, and water molecule energies of formation.

The interaction energy between the amino acid cage and water cluster within the STmod unit cell can be estimated as

$$
E_{C}=E_{\text {STmod }}-\left(E_{0}+E_{n \mathrm{H}_{2} \mathrm{O}}\right),
$$

where $E_{n \mathrm{H}_{2} \mathrm{O}}$ is the energy of the cluster composed by $n$ water molecules. Our definition differs from that of Ref. [39] by the minus sign and normalization to $n$.

Figure 3(c) presents $E_{B}$ and $E_{C}$ for each structure. $E_{B}$ increased monotonically with $n$. Similar behavior was observed in the literature. Monotonically increasing energies as a number of water molecules for confined water in single-walled nanotubes was observed, e.g., by Wang et al. [39]. External work needs be done to introduce the water clusters inside the amino acid cage since $E_{B}>0$. Thus, proteins loci with symmetry similar to $C_{n}$ structure are not viable places to catalyze the creation of water clusters. Even so, these cages are feasible places for the insertion of water clusters. The solvation of the $C_{1}$ structure $\left(C_{1 s}\right)$ does not contribute to decreasing $E_{B}$. The $D$ structure containing one water molecule $\left(D_{1}\right)$ presented the lowest $E_{B}$. One concludes this compact structure is the viable one to trap $\mathrm{H}_{2} \mathrm{O}$ molecules.

The coupling energy $E_{C}$ for $C_{n}$ structures [Fig. 3(c)] was found to be close to zero independent of the water content, except for $n=4$ and 8 . We found the preferred cluster structure to pack water molecules was the $D_{1}$ one. Tetrahedral $C_{4}$ and icosahedral $C_{8}$ water clusters appeared to have the greatest positive $E_{C}$. Thus, under basal conditions these clusters will be weakly interacting with the $C_{0}$ cage, which implies in greater mobility. The coupling energy for the other $C_{n}$ structures were $\sim 1 \mathrm{meV}$, falling into the energy scale of molecular vibrations. The $D_{1}$ structure was the one more strongly coupled to the $D_{0}$ structure.

Properties of water clusters are fundamental for understanding water in biological systems [40]. Electrons between biomolecular redox sites involve electron tunneling through the intervening space, which can be facilitated, in part, by aqueous proton movement in the reverse direction. The rate of transfer depends on both the extent and the structure of the intervening space. In particular, proteins have much of their properties governed by interactions with water [1]. These, for example, establish the free-energy landscape that determines their folding, structure, stability, and activity [41]. Structured water clusters near redox cofactors accelerate electron transfer by producing strongly coupled tunneling pathways between the electron donor and acceptor. More generally, water molecules can facilitate or disrupt this process through the degree of alignment of their dipoles and their capability to form water wires [1,7].

The calculated dipole moments $(\mu)$ are shown in Fig. 4. $C_{1 s}$ and $D_{1}$ displayed the greatest and lowest dipole moment, respectively. The point charge $q$ and $\mu$, average interaction potential is given by

$$
U=-k^{2} \frac{q^{2} \mu^{2}}{3 k_{B} T r^{4}},
$$

where $k$ is the Coulomb constant, $T$ is the temperature, and $r$ the distance between the point charge and $\mu$. From Fig. 4 and Eq. (4) one concludes a moving electron will be more strongly bound to the $C_{1 s}$ structure. The $D_{1}$ structure will trap traveling electrons more weakly, favoring electron and small ion transport. From these qualitative clues one expects sites with $C$ symmetry to be the preferred ones to host physiological processes, where large water clusters and strongly bonded electrons are needed. The abundance of free tetrahedral water in tumor tissue may be interpreted with our model framework considering that solid tumors have a tendency to enhance the permeability and retain large macromolecules $(>40 \mathrm{kDa})$ [42]. On the other hand, events where electron transport associated with lower hydration levels are the rule, will take place on loci with $D$ structure. These results confirmed the hypothesis that the structure

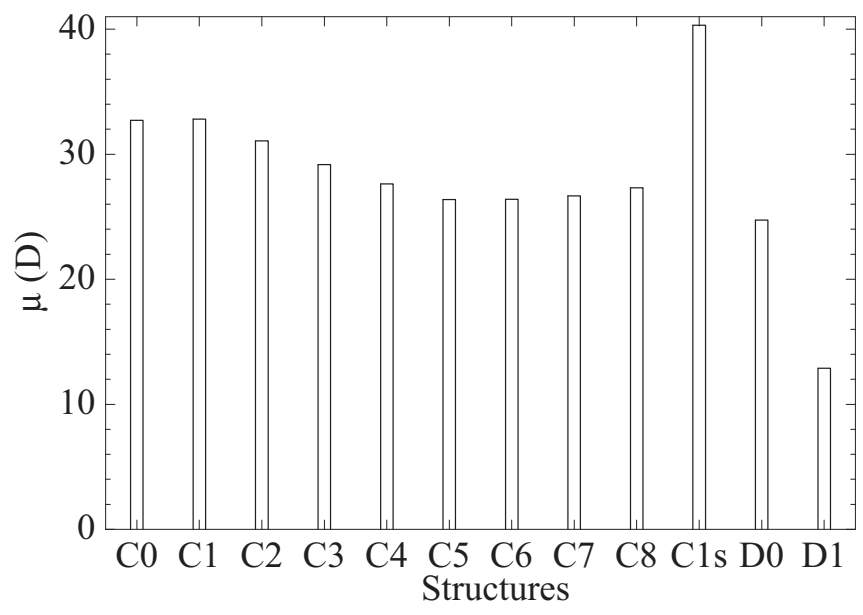

FIG. 4. Total dipole moment $(\mu)$ for each structure. 


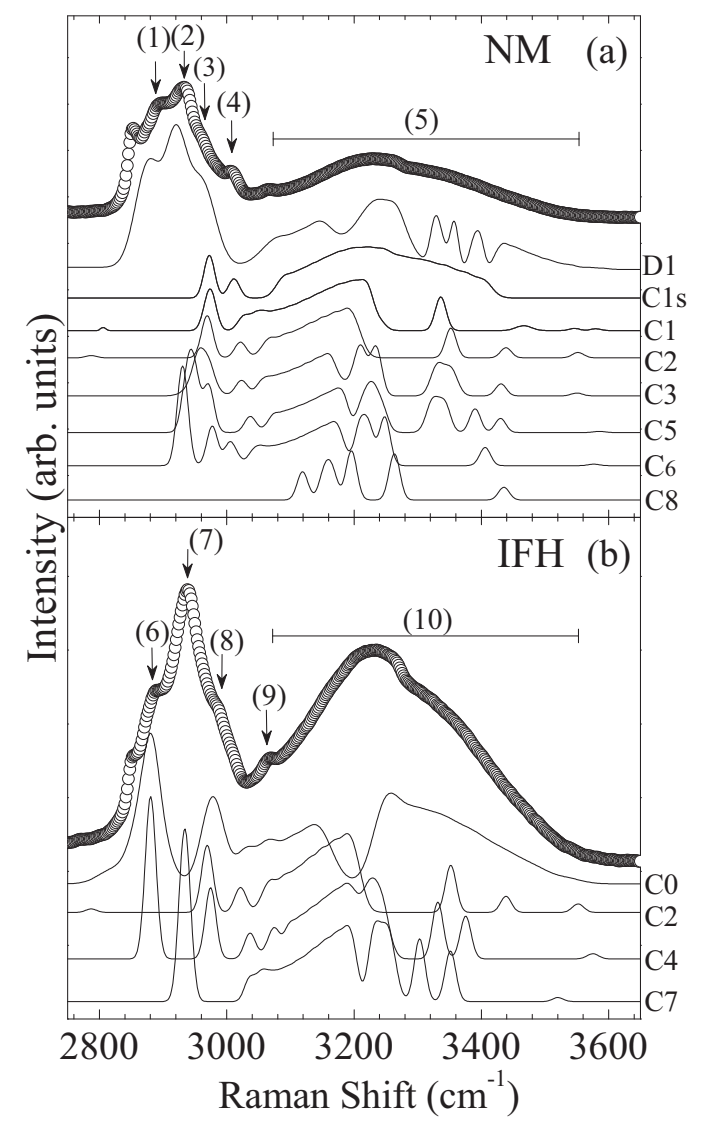

FIG. 5. Raman spectra obtained for each confinement and the experimental curves of normal (NM) (a) and inflammatory fibrous hyperplasia (IFH) (b) tissues. Numbers (1) to (10) indicate the vibrational bands discussed in the text.

of confined water alters in pathological tissues. The inflammatory response would be specially influenced by this fact.

Figure 5 presents the experimental Raman spectra of normal [NM, Fig. 5(a)] and inflammatory [IFH, Fig. 5(b)] oral mucosa tissues, compared to simulated spectra from STmod models. More experimental details concerning these data can be found in Ref. [3] It is important to notice all experimental bands or sub-bands where reproduced, except the one at $2840 \mathrm{~cm}^{-1}$. This band is usually attributed to symmetric methyl group stretching of lipids, and STmod did not take into account the presence of lipids. The NM spectrum presented strongest bands at $2933 \mathrm{~cm}^{-1}$ (band 2) that superposes two asymmetric shoulders at 2880 (band 1) and $2965 \mathrm{~cm}^{-1}$ (band 3). The left shoulder and the strongest peak were well reproduced by the $D_{1}$ vibrational modes. $C_{5}$ and $C_{6}$ contributed to the maximum at $2933 \mathrm{~cm}^{-1}$. All clusters contributed to the right shoulder, except $C_{8}$ one. The band at $3010 \mathrm{~cm}^{-1}$ (band 4) was found to be a signature of NM tissue since it was absent on IFH. This band is a convolution of contributions from $C_{1 s}, C_{2}, C_{3}$, and $C_{6}$ clusters. IFH tissue [Fig. 5(b)] was characterized by the presence of the strongest band at $2938 \mathrm{~cm}^{-1}$ (band 7) and two symmetric shoulders at 2884 (band 6) and $2992 \mathrm{~cm}^{-1}$ (band 8 ). These bands were narrowed compared to NM ones. The vibrational contributions from $C_{0}, C_{4}$, and $C_{7}$ were enough to explain this region since their Raman spectra did not present the NM signature band at $3010 \mathrm{~cm}^{-1}$. The $3070 \mathrm{~cm}^{-1}$ (band 9) band was found to be a signature of IFH tissue and only the $C_{4}$ and $C_{2}$ clusters presented this vibrational mode. Finally, the broad $\mathrm{OH}$ vibrational spectral window between 3070 and $3600 \mathrm{~cm}^{-1}$ (bands 5 and 10) appeared to be a complex superposition of contributions from $D_{1}, C_{1 s}$, $C_{1}-C_{3}, C_{5}, C_{6}, C_{8}$, and $C_{0}, C_{4}, C_{7}$ clusters for NM and IFH tissues, respectively.

\section{CONCLUSION}

Protein-water interactions are known to play a critical role in the function of several biomacromolecular systems including collagen tissues [43]. Small changes in structure and dynamical behavior of water molecules at the peptidewater interface can effectively change both the structure and dynamics of the protein function [44]. Thus, it was realized that the presence of different water clusters plays an important role in collagen properties.

The properties of the structures proposed within the STmod framework enabled us to qualitatively correlate it to some important trends observed experimentally in inflammatory tissues while keeping the structure to a minimum. We found that a tetrahedral $C_{4}$ structure is weakly bound to the amino acid cage, being it the smallest stable structure containing water clusters. The $E_{C}$ discussion lead us to conclude that tetrahedral water inside this structure has high mobility. This correlates with literature findings reporting occurrence of tetrahedral water in pathological tissues. The Raman data analysis also corroborated these findings. These clusters are the loci of possible biochemical reactions in the inflammatory cascade where strongly bound electrons play a role. We also found that $D$-like cages with one water molecule trap electrons more strongly and are thus suitable sites for water and small ion transport.

Moreover, improvements need to be implemented into the STmod to describe others tissues. For example, small glycerol or phospholipid molecules can be inserted surrounding the unit cell to mimic the brick-and-mortar-type structure of stratum corneum layer [45,46] of skin. A bidimensional STmod with lipids in one side would also be realized to simulate the dermis-hypodermis interface properties. Agents as inflammatory mediators or specific phospholipids can also be inserted in the structure and events as, e.g., inflammatory conditions, emulsifier or moisturizing effects, could be studied by molecular dynamics.

In summary, the STmod is a very promising model for more complex physical and biochemical properties calculations of tissues. Water or other biofluids microperfusion, Young's modulus at microscale, electron and proton transport, optical and electronic spectra, vibrational (Raman and IR) spectra are some examples of properties which could be calculated using this model.

\section{ACKNOWLEDGMENTS}

The authors are grateful to the Brazilian agencies Fundação de Amparo à Pesquisa do Estado de São Paulo (FAPESP), Coordenação de Aperfeiçoamento de Pessoal de Nível Superior (CAPES), and Conselho Nacional de Desenvolvimento Científico e Tecnológico (CNPq) for financial support and Multiuser Central Facilities of UFABC and CENAPAD-SP for experimental and computational support, respectively. 
[1] M. Chaplin, Water: The Forgotten Biological Molecule (Pan Stanford Publishing Pte. Ltd., Singapore, 2011), Chap. The structure of water molecule.

[2] S. Leikin, V. Parsegian, W.-H. Yang, and G. Walrafen, Proc. Nat. Acad. Sci. USA 94, 11312 (1997).

[3] L. F. C. S. Carvalho, E. T. Sato, J. D. Almeida, and H. S. da Martinho, Theor. Chem. Acc. 130, 1221 (2011).

[4] Z. Movasaghi, S. Rehman, and I. U. Rehman, Appl. Spectrosc. Rev. 42, 493 (2007).

[5] H. Cybulski and J. Sadlej, Chem. Phys. 342, 163 (2007).

[6] G. McIntyre, Med. Hypotheses 66, 518 (2006).

[7] M. Chaplin, Nat. Rev. Mol. Cell Bio. 7, 861 (2006).

[8] H. Martinho, Cancer Cares, Treatments and Preventions (iConcept Press Ltd., Hong Kong, 2013), Chap. Advances in Ramanbased optical biopsy.

[9] M. Gniadecka, H. Wulf, N. N. Mortensen, O. F. Nielsen, and D. H. Christensen, J. Raman Spectrosc. 28, 125 (1997).

[10] L. Xie, L. Xie, S. L. Kinnings, and P. E. Bourne, Annu. Rev. Pharmacol. Toxicol. 52, 361 (2012).

[11] M. O. Steinhauser and S. Hiermaier, Int. J. Mol. Sci. 10, 5135 (2009).

[12] A. Yazid, N. Abdelkader, and H. Abdelmadjid, Appl. Math. Model. 33, 4269 (2009).

[13] D. Lü, X. Liu, Y. Gao, B. Huo, Y. Kang, J. Chen, S. Sun, L. Chen, X. Luo, and M. Long, PloS one 8, e74563 (2013).

[14] R. B. Groves, S. A. Coulman, J. C. Birchall, and S. L. Evans, J. Mech. Behav. Biomed. Mater. 18, 167 (2013).

[15] N. Ren, J. Liang, X. Qu, J. Li, B. Lu, and J. Tian, Opt. Express 18, 6811 (2010).

[16] C. Zhu and Q. Liu, J. Biom. Opt. 18, 050902 (2013).

[17] I. G. Mogilner, G. Ruderman, and J. Grigera, J. Mol. Graphics Modell. 21, 209 (2002).

[18] A. D. Simone, L. Vitagliano, and R. Berisio, Biochem. Biophys. Res. Commun. 372, 121 (2008).

[19] D. L. Bodian, R. J. Radmer, S. Holbert, and T. E. Klein, in Pacific Symposium on Biocomputing (World Scientific, Singapore, 2011), pp. 193-204.

[20] I. Streeter and N. H. de Leeuw, Soft Matter 7, 3373 (2011).

[21] E. Surez, N. Daz, and D. Surez, J. Chem. Theory Comput. 5, 1667 (2009).

[22] R. A. Freitas, Nanomedicine, Vol. I: Basic Capabilities (Landes Bioscience, Georgetown, TX, 1999), Chap. 3, Tables 3-1;3-2.

[23] J. Bella, B. Brodsky, and H. M. Berman, Structure 3, 893 (1995).
[24] W. L. Jorgensen, J. Chandrasekhar, J. D. Madura, R. W. Impey, and M. L. Klein, J. Chem. Phys. 79, 926 (1983).

[25] D. J. Wales and M. P. Hodges, Chem. Phys. Lett. 286, 65 (1998).

[26] C. R. VanderSchee and K. J. Ooms, J. Phys. Chem. B, 118, 3491 (2014).

[27] N. B. Kavukcuoglu, Q. Li, N. Pleshko, and J. Uitto, Matrix Biol. 31, 246 (2012).

[28] J. Wang, P. Cieplak, and P. A. Kollman, J. Comp. Chem. 21, 1049 (2000).

[29] T. A. Halgren, J. Comput. Chem. 20, 720 (1999).

[30] M. D. Hanwell, D. E. Curtis, D. C. Lonie, T. Vandermeersch, E. Zurek, and G. R. Hutchison, J. Cheminfor. 4, 1 (2012).

[31] M. J. F. et al., Gaussian 03, Revision C.02 (Gaussian, Inc., Wallingford, CT, 2004).

[32] P. Hohenberg and W. Kohn, Phys. Rev. 136, B864 (1964).

[33] W. Kohn and L. J. Sham, Phys. Rev. 140, A1133 (1965).

[34] Cpmd, http://www.cpmd.org/, copyright IBM Corp. 1990-2008, copyright mpi fr festkrperforschung stuttgart, 1997-2001.

[35] C. Lee, W. Yang, and R. G. Parr, Phys. Rev. B 37, 785 (1988).

[36] O. A. von Lilienfeld, I. Tavernelli, U. Rothlisberger, and D. Sebastiani, Phys. Rev. B 71, 195119 (2005).

[37] I.-Chun Lin, M. D. Coutinho-Neto, C. Felsenheimer, O. A. von Lilienfeld, I. Tavernelli, and U. Rothlisberger, Phys. Rev. B 75, 205131 (2007).

[38] Y. J. Park, Y. K. Kang, B. J. Yoon, and M. S. Jhon, B. Korean Chem. Soc. 3, 50 (1982).

[39] L. Wang, J. Zhao, F. Li, H. Fang, and J. P. Lu, J. Phys. Chem. C 113, 5368 (2009).

[40] J. Gelman-Constantin, M. A. Carignano, I. Szleifer, E. J. Marceca, and H. R. Corti, J. Chem. Phys. 133, 024506 (2010).

[41] B. Halle, Philos. T. R. Soc. London. B 359, 1207 (2004).

[42] J. Fang, H. Qin, H. Nakamura, K. Tsukigawa, T. Shin, and H. Maeda, Cancer Science 103, 535 (2012).

[43] N. N. Fathima, M. Baias, B. Blumich, and T. Ramasami, Int. J. Biol. Macromol. 47, 590 (2010).

[44] T. Lima, E. Sato, E. Martins, P. Homem-de Mello, A. Lago, M. Coutinho-Neto, F. Ferreira, C. Giles, M. Pires, and H. Martinho, J. Phys. Condens. Matter 24, 195104 (2012).

[45] J. A. Bouwstra, G. S. Gooris, J. A. van der Spek, and W. Bras, J. Invest. Dermatol. 97, 1005 (1991).

[46] M. Heisig, R. Lieckfeldt, G. Wittum, G. Mazurkevich, and G. Lee, Pharm. Res. 13, 421 (1996). 